\title{
Exploring Other-Than-Human Identity: A Narrative Approach to Otherkin, Therianthropes, and Vampires
}

\author{
Clive Baldwin
}

St. Thomas University, Canada

\section{Lauren Ripley}

St. Thomas University, Canada

DOI: http://dx.doi.org/10.18778/1733-8077.16.3.02

\section{Keywords:}

Otherkin; Therians; Vampires; Narrative Identity; Spiritual Identity

\begin{abstract}
Drawing on in-depth, narrative interviews with 24 self-identified Otherkin, Therianthropes, and Vampires, we explore how members of these communities navigate Bamberg's three "dilemmatic spaces" or tensions of continuity/change, similarity/difference, and person-to-world/world-to-person fit. With regard to the first, we identify four aetiological narratives (walk-ins, reincarnation, trapped soul, and evolutionary soul), and discuss stories of shifts and awakening. For the second, we discuss how participants manage the similarity/difference tension with regard to themselves and humans, and explore categorical and renunciatory othering within the communities. Finally, we explore the ways in which members of the communities experience a barren narrative environment, and ways they seek to construct storyworlds and narrative resources as frames for establishing their identities.
\end{abstract}

Clive Baldwin is a Professor of Social Work and Canada Research Chair in Narrative Studies at St. Thomas University, New Brunswick, Canada. His research interests focus around the issue of narrative identity, spirituality, and personhood.

email address: baldwin@stu.ca
Lauren Ripley, (BA Hons), is a student of Social Work and co-researcher with Dr. Baldwin at St. Thomas University, New Brunswick, Canada. Her research interests focus around narrative identity and spirituality.

email address: hlhnd@stu.ca 


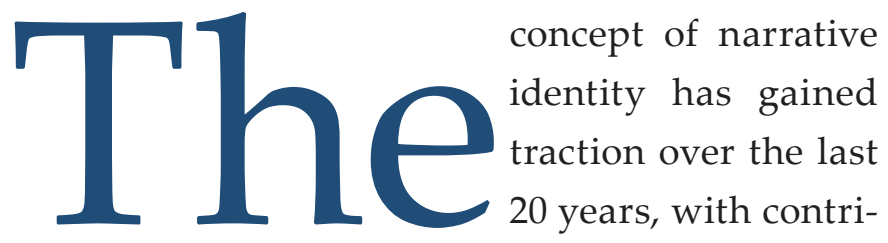

butions from psychology (e.g., Schechtman 1996), sociology (Somers 1994; Ezzy 1998), philosophy (Dennett 1992; Ricoeur 1992), social work (Baldwin 2013; Baldwin and Estey 2014), and literary theory (de Peuter 1998) to give an indicative, not comprehensive list. One of the more applied approaches in this area is Bamberg's (2004; 2010) conception of narrative identity, which consists of navigating three "dilemmatic spaces" or tensions: that of continuity/change in which the individual must make sense of her-/himself in terms of to what degree is $s /$ he the same as $s /$ he was, and to what degree different; that of similarity/difference in which the individual makes claims to being the same as others and claims to being different; and person-to-world/world to person fit, in which the individual has to account for, accommodate, or change her/his stories in the light of the stories told about her/him by others, and/or have the world account for, accommodate, or change its stories about the individual. In this article, which is based on and extends a paper presented at the Qualitatives 2018 conference at St. Thomas University, Fredericton, NB, we utilize Bamberg's model of narrative identity to develop our understanding of multiple non-standard identities, those of spiritually-oriented Otherkin, Therianthropes, and Vampires. Drawing on qualitative interviews with a range of Otherkin and Therianthropes, as well as Vampires, we will explore how such individuals construct their identities through narrative techniques. In so doing, we will argue that individuals identifying as other-than-human are simply deploying the same techniques as those with more standard identities.
We will proceed in the following manner. After some initial comments about the three communities which form the focus of this study, a note on Otherkin and Therianthropes, and a brief survey of the (somewhat scant) academic literature, we will lay out some of the narrative concepts required to understand the process of constructing and maintaining a viable and stable narrative identity. Following this, we will provide some detail about the study, the participants, and the methods. Turning to the body of the paper, we will explore how participants navigate Bamberg's three dilemmatic spaces, paralleling participant approaches to this with the construction of a more mainstream spiritual identity on the part of one of the authors [CB]. In conclusion, we will suggest that individuals identifying in part or completely as other-than-human are engaging in the same process of narrativizing identity as those with more standard identities, though are doing so with far fewer narrative resources. Ways in which Otherkin and Therian communities are addressing this will be discussed.

\section{A Note on Three Communities}

Given the lack of recognition and understanding of the three communities in this study, a note on those communities may be necessary in order to locate the individual experiences of participants in a wider narrative of Otherkinity, Therianthropy, and Vampirism. The development of collective narrative resources, and the experience of individuals who "awaken" to their otherness (see below), are intertwined, and to understand one, it is necessary to understand the other.

\section{The Otherkin Community}

The most detailed history of the Otherkin community can be found in Scribner's (2012) Otherkin 
Timeline. The community has its origins in the early 1970s when a group identifying themselves as elves came together as the Elf Queen's Daughters (EQD). Throughout the '70s and '80s the EQD published information and resources concerning the elfin life and seems to have been the main source of elfin activity. The first use of the term "Otherkind" (with a final d) was in 1990 in the Elfkind Digest, with the appearance of "Otherkin" later that year in a listserv as a way of including non-elves in the developing community. Since then, the Otherkin community, while not solely an Internet-based community, has certainly grown up alongside advances in Internet technology (Shane 2014). The Otherkin community, however, is loosely organized and appears as somewhat of a "loose and baggy monster," with all the creativity, openness, and messiness that implies. Although there are several websites for Otherkin (the most notable currently being Otherkin. net and Kinmunity.com), these are the projects of individuals rather than the result of any collective or organization. Such sites provide opportunities for Otherkin of all kin-types to discuss, share, and debate individuals and community issues, and as a repository for the growing self-understandings of the community.

\section{The Therian Community}

According to theriantimeline.com, the modern Therian community is a product of the last decade of the $20^{\text {th }}$ century, with the term "therian" becoming more commonplace after 2000 (see: http://theriantimeline.com/therianthropy/history_of_therianthropy_and_the_therian_community). The online Therian community emerged from horror media discussion boards such as Alt Horror: Werewolf, where in the early ' 90 s discussions of real Werewolves arose. While the Therian community has grown and strengthened through the Internet, it is important to note that there are older Therians, born before the advent of the Internet, who form a stable, offline community. Many Therians, it appears, initially were members of Otherkin communities (and, of course, some still identify as both), but there has been an emergent categorical distinction between the two over the years. Some communities, such as Kinmunity.com, provide a home for Therians, while there are Therian-specific communities such as therian-guide.com and http://therian-wilderness. proboards.com/.

\section{The Vampire Community}

As with the Otherkin and Therian communities, the Vampire community grew and strengthened in the '90s, though there had been, according to Browning (2014), a disjointed network of self-identifying real vampires for a couple of decades prior to this, connecting through fan clubs, Goth clubs, conventions, and fetish sites. By the "70s Vampirism became the focus of bodies such as the Vampire Research in Britain (1970), the Vampire Research Center in New York (1971), and the Vampire Studies Society (1977). In 1978, Eric Held formed the Vampire Information Exchange and published a newsletter from then until the mid-2000s. The first indication that this loose network constituted a form of community came in 1997 on Sanguinarius' Vampire Support Page (http:// www.sanguinarius.org/). Of the three communities, it seems that the Vampire community has the strongest offline presence, with organized groups in many places. Compared with the Otherkin community, the Vampire community appears relatively well-organized with recognizable "leaders" (in the sense of established and respected individuals) and some degree of structure (for example, these vampire "leaders" gather to discuss issues facing the 
community)-though the robustness of this organization should not be overstated. Unlike the other two communities, the Vampire community has been the subject of a large research study, undertaken by Suscitatio Enterprises, the results of which are available at: http://www.suscitatio.com/.

\section{A Note on Otherkin and Therians}

Although we will present further detail on the multiple distinctions to be made among Otherkin and Therians, and between Otherkin and Therians and other non-human identities; at the outset, it may be helpful to provide at least some basic definitions. Otherkin are those who identify, in whole or in part, as other-than-human. There are many different "kin" who fall under the Otherkin umbrella-dragonkin, angelkin, elvenkin, unicornkin, and so on. Therians are those who identify, in whole or in part, as other-than-human, but as Earth animals such as wolves, dogs, cats, cheetahs, and so on. For some, Therianthropy may be seen as a subset of Otherkinity, though, for others, there is a substantive distinction to be made between those who identify as mythical or fictional creatures and those who identify as earthly ones.

A further distinction pertinent to this paper is that between Otherkin and Therians, who identify as such from a spiritual basis - that is, those who view themselves as having the soul of an other-than-human being albeit within a human body ("You get a lot of [uh] Otherkin who talk about, you know, what the, the soul that's Otherkin the meat suit that's human" Participant F05)-and those whose identification is purely psychological.

Otherkin and Therians do not consciously create the identity, rather it is something present at birth, eventually realized as they "awaken" to their true identities. In this way, some participants compare it to transgender identity, where one feels and identifies with the gender opposite to that which they were designated at birth (see: Grivell, Clegg. And Roxburgh 2014). However, instead of identifying with a different gender, one identifies with a species other than Homo sapiens. The animal, or creature, that one establishes as a part of their soul is referred to as the kin type, or theriotype.

The Otherkin/Therian communities are distinctive in their heterogeneity. The Otherkin community itself expresses this through "categorical othering"non-judgmental categorization of individuals into kin type (see: Bricker 2016) with separate kin-types for and listings for members to join and, hopefully, gain support. Similarly, there are many explanations provided for Otherkinity/Therianthropy-of which later-ranging from genetics to reincarnation. The two communities are also very diverse in terms of age-the participants in our study ranging from 1771. This diversity in age is not always apparent in the literature where it is often younger Otherkin/ Therians who are the subject of study.

Finally, for the most part, these communities have not had a place within mainstream society, are subject to ridicule and "trolling" on their community websites, and thus often prefer to remain anonymous, being wary of the attention of researchers. Thus, the experience of being Otherkin or Therian remains in great part unknown.

\section{A Note on the Literature}

The literature on Otherkin, Therians, and Vampires is relatively sparse. Much of the literature focuses on the phenomena of Otherkinity and Therianthro- 
py (see, for example, Shane 2014; Cusack 2016) with only a handful focusing on the experience of Otherkin and Therians themselves (see: Grivell et al. 2014; Bricker 2016; Gagliardi 2016). While these studies are, to some degree, helpful in their descriptions of such processes as "awakening" and "shifting" (see later), the authors of these studies of experience acknowledge methodological limitations, whether in sampling or in data collection. Even Grivell and colleagues' (2014) informative and important study focused on only five participants, a sample within the parameters of their chosen approach of Interpretative Phenomenological Analysis (IPA) and one that met the requirements of IPA, which is that the sample should be homogeneous (Therian, relatively close age-range, theriotype), but in doing so is, we think, too small to encompass the diversity of the Therian community. Similarly, Bricker (2016) interviewed only seven participants and Gagliardi (2016) relied on vlogs rather than directly interviewing Otherkin and Therians. Laycock's (2012) analysis of secondary data taken from "The Vampire and Energy Work Research Study" (VEWRS), undertaken between 2006-2009, provides a useful background and information about Otherkin and Therians based on qualitative responses of 70 participants. From this data, Laycock explores the heterogeneity of the Otherkin community. However, the data from these 70 participants amounted to only 81 pages, a relatively small dataset given that these were responses to a five-part question, and thus, perhaps, lacks the rich, nuanced data required for in-depth analysis of identity-work.

In addition to some of the methodological limitations of studies of Otherkin and Therians, there are several misconceptions or understandings evident in the literature. The first of these is that of the distinction between Otherkin and Therians who view their "otherness" as having a spiritual basis and those who see it as a psychological identification. Some authors (see: Johnston 2013) do recognize this but, others seem to view Otherkin as a new, invented, or fictional religion or falling almost exclusively within a spiritual framework (see: Kirby 2005; 2008 [updated 2012]; 2009; Robertson 2013; O'Callaghan 2015; Cusack and Kosnak 2016). Laycock (2012) is less inclined to categorize Otherkin as a New Religious Movement, and, indeed, indicates that some Otherkin do not identify as spiritual individuals. But, he states that it is clear that they form what Bainbridge and Stark (1980) call an "audience cult," a "movement which supports novel beliefs and practices, but without a discernible organization" (Laycock 2012:73) which competes, albeit obliquely, with religious traditions. In contrast, in recruiting for the study reported here, it became apparent that those Otherkin and Therians identifying as such on a spiritual basis are, in fact, in the minority in their respective communities, confirming White Wolf's (2014) informal survey that indicated only about $1 / 3$ of Therians identified as such on the basis of spirituality.

A second misconception, reported by the participants in our study, is that the Otherkin and Therian communities are primarily online communities (see, for example, Robertson 2013; Shane 2014). This is understandable when the Internet is the primary method of recruiting for studies of Otherkin/ Therians, but, while there is indeed a lot of online activity, especially among younger members of the communities, older Otherkin/Therians reported that they rarely participate in the online community, preferring to maintain personal social contact with others. To view Otherkinity/Therianthropy then as an Internet phenomenon is thus somewhat misleading. 
A third misconception is to bracket together Otherkin and Therians with Furries or Bronies. The misconception here is two-fold: first, while Otherkinity and Therianthropy are involuntary identifications, Furriness is a voluntary choice; second, Furs do not see their Furriness in the ontological way Otherkin and Therians do-they see their Furriness as an assumed identify (for whatever purposes). There is, of course, some overlap for some members of each community, and some members of the Otherkin/Therian communities have been members of the Fandom at times, in particular when they are seeking to make sense of their experience of being Other. However, in general terms the Otherkin/ Therian communities separate themselves from the Fandom.

\section{The Current Study}

The data presented here on Otherkin, Therians, and Vampires form a subset of data gathered for a wider study on spiritual identity. The study has recently received funding from the Social Sciences and $\mathrm{Hu}-$ manities Research Council under the Insight Development Grant program and has been reviewed and approved by the Research Ethics Board of St. Thomas University.

The purpose of the study is to explore how individuals see and express their spiritual identity through the stories they tell, and the stories that are told about them, with a view to not only understanding the experience of non-traditional or non-mainstream spirituality (terminology is very difficult here as all currently accepted terms seem to carry with them some tendency to marginalize these experiences), but also to explore how these experiences can be brought into the wider discourse of spirituality through inter-faith activity.
Otherkin, Therians, and Vampires were sought via postings and contacts on multiple websites and Facebook pages, with others being recruited by word of mouth from earlier participants. Inclusion criteria were that participants should have capacity to provide consent, be over the age of 16 (or 18 for participants coming from one particular community - a requirement of being permitted to post to that community), define themselves as Otherkin, Therian or Vampire on the basis of spirituality, or that being Otherkin, Therian or Vampire was an important aspect of their spirituality.

Narrative-based interviews (see: Mishler 1986; Anderson and Kirkpatrick 2016) were undertaken. Most interviews were conducted via Skype or telephone with a few being conducted via Discord. A few interviews were conducted via text chat at the behest of the participants who indicated that they felt more comfortable and more able to express themselves properly this way. Interviews focused on the participants' spiritual journeys, their relationships, their engagement with the Otherkin/ Therian and other communities, representations of those communities in the wider world, and their thoughts and experiences of common spiritual concepts such as fate, serendipity, mystery, the afterlife, and so on. The interview schedule was designed so as to elicit data on sense and meaning making, identity formation over time, and the interaction between personal stories and those circulating in the wider world.

Narrative methods for understanding identity have developed over the two decades or so-with major contributions from authors in the field of psychology such as Schechtman (1996), Bamberg (2004; 2010), and, more philosophically, Ricoeur (1992) and Dennett (1992). The usefulness of narrative in 
the study of identity lies in its ability to encompass the inner stories of individuals as they seek to make sense of experience, and bring meaning to that experience (see, for example, Crites [1971] and Carr [1991] who argue that experience in and of itself bears a narrative quality), the outer stories of others that help shape experience and inner stories (see: Kenyon and Randall 1997), and meta-narratives - stories circulating in society that facilitate or hinder the construction of viable, acceptable, and stable narrative identities (see: Nelson 2001). In this paper, we explore the stories told by participants utilizing Bamberg's model of narrative identity which focuses on how individuals navigate three "dilemmatic spaces." The first of these addresses the dynamic of continuity and change in which the individual must make sense of her-/ himself in terms of to what degree is s/he the same as $\mathrm{s} /$ he was and to what degree different. The second focuses on how an individual positions her-/ himself as similar to and/or different from others. The third dilemmatic space refers to the person-toworld/world-to-person fit, in which the individual has to account for, accommodate, or change her/his stories in the light of the stories told about her/him by others and/or have the world account for, accommodate, or change its stories about the individual. This model thus encapsulates both inside-out and outside-in stories (Kenyon and Randall 1997) in that it allows for the exploration and integration of internal and external storytelling (and the dynamic between them) and how identity is formed or negotiated personally and socially (see: de Peuter 1998), and an appreciation of the "narrative environment," the stories which surround us in our everyday lives (Randall and McKim 2008) which "impel, pervade, and mediate our understandings of our everyday, experiential worlds" (Parsons 2009) and within which we orient ourselves.

\section{Participants}

Here we draw on interviews with participants in the wider study who self-identify as Otherkin, Therian, or Vampire. To date (the study is ongoing) there are 24 participants included in this part of the study. As can be seen, there is a fairly even split between male and female participants, and between those identifying as Otherkin and those as Therian. The age range of participants was 17-71, with, again, reasonably even representation from younger and older generation members of the communities.

\section{Continuity/Change}

The shift from a human to a non-human identity would seem to be one that is fundamental to one's sense of self, an almost complete leaving behind of one's old identity and the immersion in another, radically different one. For Otherkin, Therians, and Vampires, however, this break with the past is less radical than it might appear as the new, authentic, identity is accommodated into a life story of past, present, and future in ways that create a sense of continuity.

First, there is the establishment of a narrative aetiology-the construction of a story that explains the origin of something, in this case, the experiences of being "other-than human." In this study, four aetiological narratives were identified: walk-in narratives, reincarnation, trapped soul, and evolving soul.

In the event of a walk-in soul, the original soul of the body is said to have departed, and thus a new soul has come in to take its place. In the case of Otherkin, and Therianthrope, this soul would be that of the animal or creature with which they identify: 


\begin{tabular}{|c|c|c|c|c|}
\hline Participant code* & Gender & Identity & Community & Self-identified as older generation \\
\hline N42 & Female & Dragon & Otherkin & \\
\hline N43 & Male & Mexican Wolf & Therian & Yes \\
\hline N44 & Male & Wolf & Therian & \\
\hline N45 & Female & Lioness/Canine & Therian & \\
\hline N46 & Female & Dragon/Coyote & Otherkin & \\
\hline N47 & Male & Dragon & Otherkin & Yes \\
\hline N48 & Male & Coastal Brown Bear & Therian & Yes \\
\hline N49 & Male & Medieval character & Otherkin & Yes \\
\hline N50 & Female & Dragon & Otherkin & \\
\hline N51 & Female & Unicorn & Otherkin & \\
\hline N53 & Female & Cheetah & Therian & \\
\hline N54 & Male & Dragon & Otherkin & Yes \\
\hline N55 & Female & Angel & Otherkin & \\
\hline N56 & Male & Medieval knight & Otherkin & \\
\hline N57 & Male & Werewolf & Therian & Yes \\
\hline N58 & Female & Werewolf & Therian & \\
\hline N59 & Female & Wolf/Siberian Husky & Therian & \\
\hline N60 & Male & Werewolf & Therian & Yes \\
\hline N61 & Male & Elf & Otherkin & Yes \\
\hline N62 & Female & Elf & Otherkin & Yes \\
\hline F05 & Male & Elf & Otherkin & Yes \\
\hline F11 & Female & Elf & Otherkin & \\
\hline $\mathrm{F} 12$ & Female & Vampire & Vampire & \\
\hline F13 & Female & Vampire & Vampire & Yes \\
\hline
\end{tabular}

* Participant codes are those used in the wider project.

I consider myself a walk-in, in that I'm not the original spirit in the body...for most people who identify that way, the spirit previously in the body left or didn't want to be there anymore. That's what happened to me. [Participant N55 Angel]

...there was a host that had been born in this body along with me and she left at nineteen, so essentially I'm kind of a walk-in... [F11 Elf]

In the case of reincarnation an individual believes that her/his past life was lived out as the animal or creature with which they identify and that following their death, s/he was reincarnated in a human body:

...but, spiritually, I believe this is like a complete reincarnation [uh] my thoughts for when I die is that God will then take my body and put it back into the body of a Mexican wolf where I can live out my life as I felt this life should have been. [Participant N43 Mexican wolf]

I also think that [um] personally as for [like] my own belief I think that [um] there are paths or we have all 
lived past lives of a sort and that [um] that affects my otherkin [um] identity. And then I believe that this particular fragment has followed me throughout [um] my lives I guess, so based on that logic I've come to conclude that there is some type of soul that gets reincarnated. [Participant N50 Dragon]

A further aetiological narrative was that of the trapped soul. This explanation discusses how an individual has accidentally been given the soul of an animal, or other non-human creature, instead of that of a human: "for whatever reason instead of receiving a human soul I got the soul of a coastal brown bear for whatever reason" (Participant N48 Coastal Brown Bear).

The final explanation which arises from a spiritual stand-point is that of evolving souls. This account expresses that the non-human soul was allocated to the human body for evolutionary purposes. An individual who ascribes to this narrative believes that in their next life s/he will live as the animal or creature with which they identify, therefore the placement of a non-human soul is used primarily for preparation. One participant stated:

...in this life and in the past life I was a human so I came back this way, but in this life I'm learning how...the qualities needed to be, to grow into what they want me to be, this is going to sound so weird [um] in the next life. [Participant N47 Dragon]

While these aetiological narratives might appear quite different, they have in common the establishment of otherness as being continuous over time-either through previous lives or as pre-existing souls. The break from human to non-human is thus ameliorated significantly (if not removed altogether). Indeed, the process of "awakening"- the process whereby individuals become aware of their other-ness-is experienced as one of authenticity.

Aetiological narratives are, however, based on prior story formulations as a means of making sense of bodily, mental, and emotional experiences. These stories are stories of "awakening" - the move from non-recognition to recognition of one's true or authentic identity. Otherkin, Therians, and Vampires, prior to awakening, are confronted with experiences for which there are few, if any, social or cultural narrative resources on which to draw in order to make sense of the experience. These experiences might be physical, such as experiencing the sensation of having a tail, or being larger than one's body:

But, feeling suddenly as though one is somewhere in the neighborhood of twenty-two feet tall can be concerning, especially if one is in a small area or driving in a car... [Participant N54 Dragon]

...for example, tail or ears that are on top of my head instead of on the side [uh] I feel routinely paw pads where my palms are, I feel them where my toes are on my feet... [Participant N44 Wolf]

I used to have phantom feelings of [like] fur on the back of my neck or ears, or a tail, but I haven't had them in a while. [Participant N53 Cheetah]

These physical experiences are framed as "phantom shifts," experiences recounted by almost all participants. It is important to note, however, that all participants emphasized that they understand these shifts as phantom-no-one subscribing to the notion of shape-shifting (a notion that is rejected on the whole in both the Otherkin and Therian com- 
munities). Thus, the continuity of the human body is maintained alongside the continuity of being Otherkin or Therian.

A second form of shift also relating to the body are what are termed "sensory shifts." These are times in which the senses become heightened, and resemble that of the kin/theriotype, these shifts usually occur alongside a mental shift. An example of this would be a wolf-kin suddenly having an increased sense of smell or hearing:

...especially it happens when I'm outside, you know, I just [um] I'll have instances where I'll be talking to someone or just in my own little world, and then suddenly I'll kind of just focus in on hearing birds and, [um] for, I'll be passing by somewhere and the, the smell will really catch me [laugh]. [Um] Just things like that. [Participant N58 Werewolf]

In addition to phantom and sensory shifts, Otherkin and Therians may experience moving into the mind-space of the kin/theriotype with whom s/he identifies, and thus $\mathrm{s} / \mathrm{he}$ will begin to act, and respond to the environment, as though s/he were that animal or creature. Such shifts are called "mental shifts" and are experienced as both desire, as in the following quotes,

I felt this yesterday, I felt the urge just to want to run on all fours, and just [uh] just become a wolf. [Participant N43 Mexican wolf]

I would have, I don't know, I would just have different moments where I would want to [like] bark or do things that canines do. [Participant N58 Werewolf]

or in focus of attention, as reported by the following participants:
I start sniffing the air and I start [uh] really observing what is around me. I start listening really close if something is around me I might start growling, whining [um] if I get depressed I might start howling [um] I, I just I begin to act more wolf like than, than human... [Participant N43 Mexican wolf]

The human aspects of me fade into the background, and the wolf aspects of me come into the foreground, so when I'm in a deep shift, I can quite literally start acting like a wolf and, and that includes things from the way my mind works, I feel like my mind takes on a completely different form, I think differently I, I interact with the world differently [um] depending on the strength of the shift. [Participant N44 Wolf]

One of the biggest problems with dragonkin is that when we're immature, we definitely let our emotions get the best of us and this can push past well into adulthood and until we learn to recognize it, it really does stifle our connections with other people because, while we think we're being rational, they see that we are clearly not. [Participant N46 Dragon/Coyote]

Another form of shifting, albeit less noted within our interviews, is emotional shifts, which occurs when an individual experiences a move towards feeling more as the kin/theriotype whilst in a particular emotional state. For instance, during times of sadness or anger, or more generally-in times of high emotions. These shifts are usually accompanied by other types of shifts, commonly mental shifts.

Eventually I began to feel [um] horns when I was [like] in certain moods, as well as a, a tail and eventually I got to feel wings... [Participant N50 Dragon] 
Finally, dream shifts are described as happening while an individual is asleep. During this time the kin/theriotype will stimulate the brain, which results in the person dreaming as if they were that kin/theriotype, many participants also mention dream shifts bringing about past life recall.

I'm just able to be a wolf in my dreams, and [um] it kind of helps with, [uh] you know, my day life, you know, like obviously can't shift into a wolf so that's kind of like my, my fun time [laughing]. [Participant N58 Werewolf]

Some participants narrativize these experiences through developing a notion of identity in which they view their nature not as dual, but as a human-kin/theriotype blend with characteristics of one or the other being foregrounded at certain times and in certain circumstances. Several participants reported that following their awakening they were able to increase their control over their shifts-intentionally willing them at times:

I can voluntarily trigger them as well, [um] but it requires concentration and time, and a meditative state, and it's sort of like [um] we used to call it getting small like [uh] there was a group of us who would get together for spiritual practices, you know, we'd, we'd get together and meditate and, you know, talking about our Therianthropy and how, you know, a group of us who were spiritual Therians, [uh] our spiritual practices and why we felt we were the way we were, and it was during those [uh] meditative practices that I kind of figured out how to get into that bear headspace... [Participant N48 Coastal Brown Bear]

So, when I am alone, my mind shifts from my human mind to my wolf mind. Since my distracting human thoughts get in the way, I sometimes have to medi- tate first. Then I just forget what it's like to be human. When this happens, I often run around on all fours and will even chase things or bite things. It hurts to run on all fours, but it feels natural. [Participant N59 Wolf/Siberian Husky]

Sometimes, I can, I can use meditation and [uh] mindfulness to induce a shift, if I want to have one I, I can make it happen, but I also have involuntary shifts, so sometimes I will get shifts and there might not be an obvious trigger or an obvious why, it just kind of happens. [Participant N44 Wolf]

Thus, while Otherkin/Therians report shifts as more or less continuous experiences, these experiences are made sense of retrospectively through the process of awakening, the stories of which serve to facilitate the transition or change in self-identity. The experiences of awakening lend some support to Boje's (2000; 2009) concept of ante-narrative, a pre-narrativized experience that requires integration into a sense-making narrative, rather than the notion from Crites (1971) and Carr (1991) that experience is narrative in nature. Participants reported wrestling with peculiar experiences for which they had few cultural narrative resources prior to finding the Otherkin/Therian communities which provided a framework for understanding their experiences, namely, stories of shifts. We shall return to the subject of the lack of cultural narrative resources for the development of non-human identities later, when we discuss Fricker's (2007) notion of hermeneutical injustice and Partridge's (2004; 2005; 2014) concept of occulture.

\section{Similarity and Difference}

Bamberg's (2010) second dilemmatic space relates to how individuals (or groups) position themselves 
as similar to, or different from, others. This space it seems is key to understanding the experience of Otherkin, Therians, and Vampires.

The first, and most obvious, tension in this space is that of involving the similarity and difference of Otherkin, Therians, and Vampires to and from humans. By definition Otherkin, Therians, and Vampires are those who identify in whole or in part as other-than-human. Nevertheless, they accept their bodies as human-for example, they do not generally accept the notion of shape shifting as a possibility and are very skeptical of those who claim to be able to do so

...teeth and the claws are a thing that I always had, but didn't [um] and they were there, they were so real and, you know, and for a while I had to close my mouth in a particular way, otherwise I was afraid, I was afraid I'd bite myself. But it, it, you know, it's not there because you're still human, you're still physically a human being as much as it, for whatever reason, might not feel that way... [Participant N42 Dragon, authors' emphasis]

and that they are required to enact their lives in a human world:

....and I am perfectly, you know, capable of, minus my anxiety and the panic attacks, capable of holding down jobs [uh] managing financial affairs, managing my life, managing friends, family, managing a very professional and [uh] human life... [Participant N43 Mexican Wolf]

...we're, [um] you know, we're members of society just like everybody else. We all have jobs and lives and families and, [um] you know, we're, we're normal, but we're not normal, but we are normal... We're just like, you know, everyday people just living our lives, just trying to survive, you know [um], having fun, just, just like everybody else, and that's what I want people being able to see, you know. [Participant N58 Werewolf]

Turning to similarities and differences between and within other-than-human communities, almost all participants referred in some way or other to those they experienced from others. Getzler (2013) discusses two forms of othering: categorical othering, that is, the demarcation of categories indicating difference without judgment or denigration and renunciatory othering, othering that excludes individuals from the group.

In participant interviews, both forms of othering were evident. Categorical othering was evident in the plethora of kin-types (and within-kin types) and theriotypes referred to, and in the Vampire community a distinction was made between sanguinarian and psychic vampires (see: Laycock 2010 for an excellent exposition of the Vampire community). This process of categorical othering is demonstrated by the general acceptance of all kin- or theriotypes, and many participants commented on the welcoming nature of the community:

...overall it's, unless you manage to get on the bad side of the community or garner a bad name for yourself through whatever your actions happen to be that deserve it are, generally pretty accepting because they know what it's like to not be accepted... so they are welcoming so long as you don't hurt them and [uh] I should hope that most people would be welcoming so long as you don't hurt them. [Participant N42 Dragon]

So the knowledge that's in the community, and the understanding and acceptance and the willingness of so many of these people to help you, yourself because they're feeling the same things they felt, the 
same things you're feeling [um] and they just want to help you gain that understanding... [Participant N42 Dragon]

This process of categorical othering is useful in that it allows for an acknowledgement of difference, and indeed individual uniqueness, within a community. However, the very identification of uniqueness in some ways might limit the support able to be provided by the community:

It's definitely, that's one of the biggest challenges with Otherkin is that we can help each other immensely, but at the same time some of us just have really different goals, and that means that we are bound to be divergent eventually. [Participant N46 Dragon/Coyote]

One participant, for example, stated:

...it's a very strong pull as I said, like there's definitely people out there that where they are I have no clue I have not been in [uh] every, I'm in virtually every Otherkin Discord, so, I'm on a lot of forums, I'm in a couple of groups on Facebook, et cetera... I look, and I look, and I look, and there's some very intense Google searching quite often [uh], but [uh] I've just never been able to find anyone, but even after a year and a half of just straight working out my life every day at least once a week...it, it's quite, it's quite lonely... 'cause no one's really in my situation. [Participant N56 Medieval knight]

Renunciatory othering was evident in some participants' comments when discussing the nature of Otherkinity/Therianthropy/Vampirism in that they made a distinction between "real" or "true" other-than-human identities and "lifestyle" identities, that is, identities assumed rather than experienced as ontological: ...now there's a lot of teenagers, and some folks age out like it's a phase [uh] [uh] it's been jokingly said the same as like [uh] Therian until graduation, you know, as a play on the, the lesbian until graduation [uh] thing [um]... [Participant N46 Dragon/Coyote]

Living Vampires I've commonly seen associated with the thought that if you buy fangs, then you're a vampire, which is, frankly, bullshit. If buying fangs makes you a vampire, then putting feathers in your hair makes you a chicken. I personally regard a vampire as being someone who needs energy and/or blood to remain physically healthy, where there is a marked improvement in their physical and mental aspects after feeding, through either of the two channels. [Participant F12 Vampire]

And, I mean, it's, it's part of the seeking of identity to whether it's, you know, there are a lot of [um] folk in, you know, people that take the identity because it's cool, it's different, it makes them unique, it makes them individual [um] and it's something that will go away in time because they're not Otherkin. They're, you know, they're putting on the, the goth outfit to fit with the tribe, dance to the music and then when they turn thirty, they'll go work for an accounting firm and, you know, everything goes back to normal... [Participant F05 Elf]

While renunciatory othering might appear as undermining the openness and acceptance within the communities, it does serve, we think, a positive function in that it helps protect the community from external criticism by focusing on the essentialist nature of the identity. Other-than-human communities have been the target of hostile and malicious attacks due to the attackers' perception of those communities and identities as being absurd. It is our opinion that focusing on the essence of other-than-human 
identities by attempting to distance oneself from transitory, assumed, or lifestyle Others this might help those whose experience of otherness is ontological in the stabilization of their identities in the face of a hostile world.

What is clear from the data is that there are multiple and nuanced variations in the experience of navigating the dilemmatic space of similarity and difference. On the one hand, similarity may help with both offering and receiving support from community members, on the other, difference is rooted in one's deep experience and cannot, and should not, be sacrificed to communal understandings. This, however, we would suggest, in a post-modern, identity-oriented world is the experience of us all.

\section{Person-to-World and World-to-Person Fit}

The third of Bamberg's dilemmatic spaces addresses the interplay between the stories of individuals and those circulating in the wider world. For most of us, while there is a certain degree of incongruence-for example, while I [CB], being Catholic, need to reconcile, or at least live with, the notion of a living God with immersion in the Enlightenment meta-narrative of progress and Reason (or postmodern relativism), being Catholic is a generally acceptable formulation of one's identity-for Otherkin and Therians, this tension is difficult to navigate as the narrative resources available to help one navigate this tension are extremely limited. For example, unlike Catholicism the Otherkin and Therian communities do not have canonical texts that lend substance and tradition to their members. Canonical texts in religious and spiritual traditions can serve the function of establishing and maintaining a stable identity in a world that does not share the same views as the religious group (see: Hammer and Rothstein
[2012:120] regarding Jehovah's Witnesses where, "The text reaches beyond mere doctrinal issues and becomes the prime marker of social and religious identity").

Canonical, para-canonical, and other community texts help establish a storyworld in which being a member of the group makes sense and is supported. For me [CB] as a Catholic, I have recourse to scripture, the Church Fathers, the Catholic tradition, and a multitude of spiritual and theological texts and other resources to draw in as I navigate my individual Catholic journey in the modern world. And because these resources are long-established, drawn on by millions of believers, and are within generally recognizable human experiences, being Catholic is relatively unproblematic both for myself and for others. For Otherkin, Therians, and Vampires, however, such resources do not exist or are so limited that understanding the experience of Otherkinity/Therianthropy/Vampirism becomes distorted, rejected, or recuperated into socially and culturally available stories such as those of mental illness, pretending, or an extreme version of egoistic attention-seeking.

This being so, Otherkin, Therians, and Vampires fall subject to what Fricker (2007:155) calls hermeneutic injustice, that is, "the injustice of having some significant area of one's social experience obscured from collective understanding owning to a structural identity prejudice in the collective hermeneutic resource." As one participant stated:

[Otherkinity is]...a mix of everything really, it's, it's a, it's a Gestalt all of this stuff a melange kind of thing [um] the problem with looking into the science of things is that [uh] humankind is wonderfully arrogant and self-centered... so if you, for example, were 
able to [um] find the, the gene that caused what's it called polydactylism the, the webbing between fingers... and say, "This, this happens here in only these people, this might be an indication of them being Otherkin," medical science would go, "No, no, no it's just a variation on human. Everything it is a variation on human." [Participant F05 Elf]

Given the lack of a collective hermeneutic resource for Otherkin, Therians, and Vampires to draw on to understand their experience, many are forced to live with doubts about the truth of their identity:

And you know what self, self, self-doubt and self-questioning is a big one for a lot of Otherkin. We all go through periods, I mean, I, I identify as an elf for, you know, probably as far as I can think, you know, I had the label for forty-five plus years, I've known I was something different from over fifty, but there are still days I look in the mirror and think, "OK. Really? What are you on? This is this nonsense. It's, dude, you're fifty-six, stop being a twelve-year-old, you know, get, get on with life, we need to make the rent, pay the bills, you know, do the adult thing..." [Participant F05 Elf]

I want to understand myself better and I want to find, one day if it's ever possible, some true affirmation that all of this is completely real and legitimate and not something that my mind just made up in a fever dream. But, that is not something I plan on ever happening. It's not a luxury that I am likely to receive, as nice as it would be." [Participant N46 Dragon/Coyote]

In response to the lack of social and cultural resources readily available to them, some Otherkin, Therians, and Vampires draw on fragments, piecing them together into a more or less coherent storyworld, which then provides a framework within which their experiences make sense. One highly articulated expression of this would be the Silver Elves, Zardoa, and Silver Flame (Participants N61 and N62), who, over decades of living as elves, and over the course of multiple publications (43 books to date, see, for example, The Silver Elves 2014; 2015) have drawn on Jungian psychology, literature, history, mythology, shamanism, and a range of other occult resources. The result is, to our minds, as coherent a storyworld as one finds. Similarly, Participant N54, constructed a dragon-focused religion (to which he has attracted a small number of followers) complete with rituals, rules of living, a calendar establishing sacred time, and so on. Failing to find sustaining resources in the "real" world, some Otherkin turn to fiction. For example, Participant N51 (Unicorn) found support for her identity through The Last Unicorn (Beagle 1968):

That might be one of the reasons why The Last Unicorn [the movie, and the book, which I read later in my life] resonates so strongly with me. "These days it takes a cheap carnival trick to make folks recognize a real unicorn" - that quote from The Last Unicorn illustrates how I feel when I walk in my city in winter with my unicorn winter hat [it is fake white fur with a horn, ears, and a rainbow mane, and it's pretty and I love it]. When I wear this hat, people call me "unicorn" and a girl once stopped me in the streets to hug me. It was nice, but also so sad and lonely, because she hugged a hat on a human, and nothing more.

Similarly, The Silver Elves (N61 and N62) draw on Tolkien's (1977) The Silmarillion; and N56, a Medieval knight, lives in the world of Malory's (1999) Le Morte d'Arthur.

Participants also drew on art, history, anthropology, and archaeology to embed the experience of Other- 
kin in a much longer story. For example, Participant N54 (Dragon):

I mean, I've found in my own research depictions of [uh] cultural depictions of dragons as far back as [uh] ten thousand years ago...I mean, the terminology that we're, we're using now is new, but the cultural idea seems to be much, much older...Well, and I don't even know if it's necessarily hidden so much as not looked at in that way. I mean, if you look at, for example, [uh] tribal or native American [societies] and some of their practices of wearing the, the skins or, you know, claws or what have you of an animal to take on that creature's traits could be seen as somewhat similar or someone, you know, born blessed by this particular animal or this particular spirit. Things like that go back in dozens if not most primitive cultures all the way up through certain modern cultures.

This process would seem to be reflective of what Partridge (2004; 2005; 2014) refers to as "occulture," that is, how everyday life and popular culture are drawn upon in order to re-enchant the world, and thus help us understand contemporary non-secular lifeworlds. The concept of occulture has been applied to Otherkin/Therian/Vampire communities by authors such as O'Callaghan (2015) and Robertson (2013). It would be interesting to explore the impact of an emerging Otherkin/Therian/Vampire fiction on the respective communities. Laycock's (2012) contribution here is important, though it is an area that would serve further, detailed, empirical exploration.

As communities, also, Otherkin, Therians, and Vampires are generating a pool of narrative resources for others to draw upon in their own meaning-making process (see, for example, the Otherkin experiences reported on sites such as https://www.wattpad. com). Although the tendency towards emphasizing uniqueness (see section on similarity and difference) may mitigate against the development of templated story-frames into which individuals might insert their personal experiences, the sharing of stories, the development of a lexicon through which to express one's experience, and the gathering of ideas, insights, and connections related to the experiences that being other-than-human have started, albeit slowly and in a small way, to change the narrative environment in which Otherkin and Therians can understand, develop, and articulate their identities.

\section{Limitations of the Study}

As indicated at the outset, the data presented here form part of a wider, ongoing study of spiritual identity-not just among Otherkin, Therians, and Vampires, but among followers and practitioners of alternative spiritualities. As such, it is a work in progress and findings are still emerging, though those presented here have been recurrent over several or more interviews. While the current study size $(\mathrm{N}=24)$ is larger than other studies reported in the literature, it is still relatively small for such diverse communities, and there are some kin-types with whom we are still to gain contact, such as otakukin, other fictionkin, griffinkin, demonkin, and so on. Thus, the findings here can only be provisional if generalized to the wider Otherkin/Therian/Vampire communities.

A second limitation lies in the recruitment methods for the study. If, as some older generation community members say, there are many older generation members of the communities who do not engage with the Internet, then there is a potential research population yet to be accessed-and a population that would be important in understanding the complexity and development of Otherkinity, Therianthro- 
py, and Vampirism. Participants in this study have offered to distribute materials to those they know, but have said that many older generation members simply want to live their lives and do not want to engage with the communities or researchers.

A third limitation is that the study, by necessity, is reliant on self-selecting volunteers, and we cannot know how representative the views of such are of the wider communities. Standard qualitative methods-such as working towards data saturationhelp here, but cannot thoroughly ameliorate the potential problem.

\section{Final Thoughts}

In this paper, we have attempted to make headway in understanding the process of narrative identity formation among members of other-than-human communities. Although identification as Otherkin, Therian, or Vampire may appear strange to the human community, the process whereby individuals

\section{References}

Anderson, Claire and Susan Kirkpatrick. 2016. "Narrative Interviewing." International Journal of Clinical Pharmacy 38(3):631-634.

Bainbridge, William S. and Rodney Stark. 1980. "Client and Audience Cults in America." Sociological Analysis 41(3):199-214.

Baldwin, Clive. 2013. Narrative Social Work: Theory and Application. Bristol: Policy Press.

Baldwin, Clive and Jennifer Estey. 2014. "The Self and Spirituality: Overcoming Narrative Loss in Aging." Journal of Religion and Spirituality in Social Work 34(2):205-222.

Bamberg, Michael G. 2004. “Talk, Small Stories, and Adolescent Identities." Human Development 47:366-369. awaken to their identities seems to be very similar, if not identical, to the process by which human identities are formed-that is, in seeking to make sense of experience, we explore potential narrative frames (see: Boje 2000; 2009). In so doing, we bring together elements of our physical, mental, emotional, cultural, and social environments. Our emergent narrative identity is tested and shaped in interaction with those environments. For those identifying as other-than-human this process is, to be sure, far more difficult in that there are not ready-made, socially acceptable identity templates to adopt. The other-than-human communities, however, are contributing to the general narrative environment through blogs, websites, chat rooms, publications, and so on, thus extending the narrative resources available to those who are exploring but have not yet stabilized their other-than-human identity. Whether the growth of these other-than-human resources will have a major impact on the acceptability of such identities awaits to be seen.

Bamberg, Michael G. 2010. "Who Am I? Narration and Its Contribution to Self and Identity." Theory and Psychology 21:1-22.

Beagle, Peter S. 1968. The Last Unicorn. New York: Viking Press.

Boje, David M. 2000. Narrative Methods for Organizational and Communication Research. London: Sage.

Boje, David M. 2009. Storytelling Organizations. Thousand Oaks, CA: Sage.

Bricker, Natalie. 2016. “Life Stories of Therianthropes: An Analysis of Nonhuman Identity in a Narrative Identity Model." BA Thesis, Department of Psychology, Lake Forest College. 
Browning, John E. 2014. "The Real Vampire Community: A Concise History." Pp. 368-370 in The Vampire in Europe: A Critical Edition, edited by M. Summers. Berkeley, CA: Apocryphile Press.

Carr, David. 1991. Time, Narrative, and History. Bloomington: Indiana University Press.

Crites, Stephen. 1971. "The Narrative Quality of Experience." Journal of the American Academy of Religion 39(3):291-311.

Cusack, Carole M. 2016. "Spirituality and Self-Realisation as 'Other-Than-Human.' The Otherkin and Therianthropy Communities." Pp. 40-57 in Fiction, Invention, and Hyper-Reality: From Popular Culture to Religion, edited by C. M. Cusack and P. Kosnak. Abingdon: Routledge.

Cusack, Carole M. and Pavol Kosnak, (eds.). 2016. Fiction, Invention and Hyper-reality: From Popular Culture to Religion. Abingdon: Routledge.

de Peuter, Jennifer. 1998. "The Dialogics of Narrative Identity." Pp. 30-42 in Bakhtin and the Human Sciences, edited by M. M. Bell and M. Gardnier. London: Sage.

Dennett, Daniel C. 1992. "The Self as a Center of Narrative Gravity." Pp. 275-288 in Self and Consciousness: Multiple Perspectives, edited by F. Kessel, P. Cole, and D. Johnson. Hillsdale, NJ: Lawrence Erlbaum.

Ezzy, Douglas. 1998. "Theorizing Narrative Identity: Symbolic Interactionism and Hermeneutics." The Sociological Quarterly 39(2):239-252.

Fricker, Miranda. 2007. “Hermeneutical Injustice.” Pp. 147-175 in Epistemic Injustice: Power \& the Ethics of Knowing, edited by M. Fricker. Oxford: Oxford University Press.

Gagliardi, Nika R. 2016. “Otherkin and Therian in the Virtual Space: Communicating Identities of the Private and Public Self." International Textile and Apparel Association (ITAA) Annual Conference Proceedings. Iowa State University Digital Repository: Iowa State University. Retrieved April 27, 2018 (http://lib. dr.iastate.edu/itaa_proceedings/2016/posters/141).

Getzler, Melanie. 2013. Othering among Otherkin: The Discursive Negotiation of the Face-Threat of Exclusionary Othering in a Demarginalizing Internet Community. MA Thesis, Department of Telecommunications, Indiana University.

Grivell, Timothy, Helen Clegg, and Elizabeth C. Roxburgh. 2014. "An Interpretative Phenomenological Analysis of Identi- ty in the Therian Community." Identity: An International Journal of Theory and Research 14(2):113-135.

Hammer, Olav and Mikael Rothstein. 2012. "Canonical and Extracanonical Texts in New Religions." Pp. 113-129 in The Cambridge Companion to New Religious Movements, edited by O. Hammer and M. Rothstein. Cambridge: Cambridge University Press.

Johnston, Jay. 2013. “On Having a Furry Soul: Transpecies Identity and Ontological Indeterminacy in Otherkin Subcultures." Pp. 293-306 in Animal Death, edited by J. Johnston and F. Probyn-Rapsey. NSW: Sydney University Press.

Kenyon, Gary M. and William L. Randall. 1997. Restorying Our Lives: Personal Growth Through Autobiographical Reflection. Westport: Praeger.

Kirby, Danielle. 2005. Fantasy and Belief: Alternative Religions, Popular Narratives and Digital Cultures. Abingdon: Routledge.

Kirby, Danielle. 2008. "Alternative Worlds: Metaphysical Questing and Virtual Community Amongst the Otherkin." Pp. 275-287 in Through a Glass Darkly: Reflection on the Sacred, edited by F. Di Lauro. Sydney: Sydney University Press.

Kirby, Danielle. 2009. "From Pulp Fiction to Revealed Text: A Study of the Role of the Text in The Otherkin Community." Pp. 141-154 in Exploring Religion and the Sacred in a Media Age, edited by C. Deacy and E. Arweck. Farnham: Ashgate.

Kirby, Danielle. 2012. "Alternative Worlds: Metaphysical Questing and Virtual Community Amongst the Otherkin." Pp. 129140 in Handbook of Hyper-Real Religions, edited by A. Possamai. Leiden: Brill.

Laycock, Joseph. 2010. “Real Vampires as an Identity Group: Analyzing Causes and Effects of an Introspective Survey by the Vampire Community." Nova Religio: The Journal of Alternative and Emergent Religions 14(1):4-23.

Laycock, Joseph P. 2012. “'We Are Spirits of Another Sort': Ontological Rebellion and Religious Dimensions of the Otherkin Community." Nova Religio: The Journal of Alternative and Emergent Religions 15(3):65-90.

Malory, Thomas. 1999. Le Morte d'Arthur. New York: Penguin Random House.

Mishler, Elliot G. 1986. Research Interviewing: Context and Narrative. Cambridge, MA: Harvard University Press. 
Nelson, Hilde L. 2001. Damaged Identities, Narrative Repair. Ithaca, NY: Cornell University Press.

O'Callaghan, Sean. 2015. "Navigating the 'Other' World: Cyberspace, Popular Culture and the Realm of the Otherkin." Culture and Religion 16(3):253-268.

Parsons, Allan. 2009 "Narrative Environments: How Do They Matter?" Rhizomes 19. Retrieved September 15, 2018 (http:// www.rhizomes.net/issue19/parsons/index.html).

Partridge, Christopher. 2004. The Re-Enchantment of the West: Volume 1 Alternative Spiritualties, Sacralization, Popular Culture and Occulture. London, New York: T\&T Clark International.

Partridge, Christopher. 2005. The Re-Enchantment of the West: Volume 2 Alternative Spiritualities, Sacralization, Popular Culture and Occulture. London, New York: T\&T Clark International.

Partridge, Christopher. 2014. “Occulture is Ordinary.” Pp. 113133 in Contemporary Esoteric, edited by E. Asperm and K. Granholm. London: Routledge.

Randall, William L. and Elizabeth McKim. 2008. Reading Our Lives: The Poetics of Growing Old. Oxford: Oxford University Press.

Ricoeur, Paul. 1992. Oneself as Another. Chicago: University of Chicago Press.

Robertson, Venetia L. D. 2013. "The Beast Within: Anthrozoomorphic Identity and Alternative Spirituality in the Online
Therianthropy Movement." Nova Religio: The Journal of Alternative and Emergent Religions 16(3):7-30.

Schechtman, Marya. 1996. The Constitution of Selves. Ithaca, New York: Cornell University Press.

Scribner, Orion. 2012. Otherkin Timeline: The Recent History of Elfin, Fae, and Animal People. Version 2.0. Retrieved March 02, 2019 (http://frameacloud.com/wp-content/uploads/2015/01/Scribner_ Timeline2p0.pdf).

Shane, Margaret. 2014. "Some People Aren't People on the Inside: Online Connectivity and Otherkin Subjectivities." Pp. 260274 in Educational, Psychological, and Behavioral Considerations in Niche Online Communities, edited by V. Venkatesh, J. C. Castro, and J. E. Lewis. Hershey, PA: Information Science Reference.

Somers, Margaret R. 1994. "The Narrative Constitution of Identity: A Relational and Network Approach." Theory and Society 23(5):605-649.

The Silver Elves. 2014. Living the Personal Myth: Making the Magic of Faerie Real in One's Own Life. USA: Self-published.

The Silver Elves. 2015. Faerie Unfolding: The Cosmic Expression of the Divine Magic. USA: Self-published.

Tolkien, J. R. R. 1977. The Silmarillion. London: George Allen and Unwin.

White, Wolf. 2014. “2013 Therian Census Results.” Presented on Werelist March 17. Retrieved September 16, 2018 (https://www. dropbox.com/s/y8vmmanknlvqpek/2013\%20TSurvey.pptx).

\section{Citation}

Baldwin, Clive and Lauren Ripley. 2020. "Exploring Other-Than-Human Identity: A Narrative Approach to Otherkin, Therianthropes, and Vampires." Qualitative Sociology Review 16(3):8-26. Retrieved Month, Year (http://www.qualitativesociologyreview. org/ENG/archive_eng.php). DOI: http://dx.doi.org/10.18778/1733-8077.16.3.02 